\title{
Commentary specifically on the article by S. Harling et al
}

Received: 15 January 2013; Accepted: 15 January 2013

\section{Dear Editor,}

Quantification of left-to-right shunting through a patent ductus arteriosus by colour Doppler is an interesting idea, but I am not quite convinced that the technique as outlined in the paper by Harling $S^{1}$ can be a reliable tool in an in vivo setting; I am aware that flow can be accurately determined by colour Doppler. $^{2,3}$ However, these studies were carried out in controlled settings (in vitro) in which the sizes, shapes, and actual velocities of the streams were known. The size of the jet, its temporal resolution, and extent of aliasing velocities can be significantly affected by the angle of Doppler interrogation, transducer frequency, and instrument settings such as gain, output power, Nyquist limit, size, and depth of the image. ${ }^{4}$ In in vivo settings, these variables vary with each patient and are highly operator dependent, which ultimately may have a negative impact on the reproducibility and accuracy of quantitative estimation. In addition, assessing the degree of shunting based on a single two-dimensional still frame can be misleading. The jets of ductal artery can be central or eccentric (wall hugging) and the sizes of the jets may vary significantly depending on the planes of interrogation. The wall-impinging jets are significantly smaller than centrally directed jets of similar haemodynamic severity, mainly because they flatten out on the wall of the main pulmonary artery. To fully appreciate the severity of shunting, one needs to examine the patent ductus arteriosus by colour Doppler in multiple sweeping planes and windows.

\section{Thieu Nguyen \\ All Children's Hospital, Pediatric Cardiology Outpatient Care Centre St. Petersburg, United States}

\section{References}

1. Harling S, Jansson T, El-Segaier M, Peronen E. Quantification of left-to-right shunt through patent ductus arteriosus by colour Doppler in children admitted for device closure. Cardiol Young 2012; 22: 57-62.

2. Tadashi T, Richard SCC. Quantitative study of steady flow using color Doppler ultrasound. Ultrasound Med Biol 1991; 17: 595-605.

3. Landwehr P, Schindler R, Heinrich U, Dölken W, Krahe T, Lackner K. Quantification of vascular stenosis with color Doppler flow imaging: in vitro investigations. Radiology 1991; 178: 701-704.

4. Stewart WJ, Cohen GI, Salcedo EE. Doppler color flow image size: dependence on instrument settings. Echocadiography 1991; 8: $319-327$ 